\title{
Atomistic Nodal Approach: a General Molecular Dynamics Method For Computing Local Thermal Conductance at Atomistic Resolution
}

\author{
Mingxuan Jiang, Juan D. Olarte-Plata, and Fernando Bresme* \\ Department of Chemistry, Molecular Sciences Research Hub, Imperial College, London \\ W12 0BZ,United Kingdom \\ E-mail: f.bresme@imperial.ac.uk \\ Phone: +44 (0)207 5945886
}

\begin{abstract}
The Interfacial Thermal Conductance (ITC) is a fundamental property of materials and has particular relevance at the nanoscale. The ITC quantifies the thermal resistance between materials of different compositions or between fluids in contact with materials. Furthermore, the ITC determines the rate of cooling/heating of the materials and the temperature drop across the interface. Here we propose a method to compute local ITCs and temperature drops of nanoparticle-fluid interfaces. Our approach resolves the ITC at the atomic level using the atomic coordinates of the nanomaterial as nodes to compute local thermal transport properties. We obtain high-resolution descriptions of the interfacial thermal transport by combining the atomistic nodal approach, computational geometry techniques and "computational farming" using NonEquilibrium Molecular Dynamics simulations. We illustrate our method by analyzing various nanoparticles as a function of their size and geometry, targeting experimentally relevant structures like capped octagonal rods, cuboctahedrons, decahedrons, rhombic
\end{abstract}


dodecahedrons, cubes, icosahedrons, truncated octahedrons, octahedrons and spheres. We show that the ITC of these very different geometries can be accurately described in terms of the local coordination number of the atoms in the nanoparticle surface. Nanoparticle geometries with lower surface coordination numbers feature higher ITCs, and the ITC generally increases with decreasing particle size.

\section{Introduction}

Nanoparticles (NPs) have garnered increasing attention due to their wide range of applications in medicine, materials science and catalysis,${ }^{1-5}$ stemming from the significant dependence of their physicochemical properties with size and shape. In addition, metallic nanoparticles have attracted considerable interest due to their efficient conversion of light into heat, which provides unique opportunities to generate and control temperature fields at the nanoscale. ${ }^{6}$

Nanoparticle heating is important in material characterization, for instance, in diffraction studies, where the intense illumination using x-ray probes can trigger nanoparticle melting in ps to ns timescales. ${ }^{7-9}$ Atomistic modelling has proven particularly helpful to address the limitations of continuum theory to describe thermal transport processes involving a phase change. ${ }^{10,11}$ The unique capabilities of nanomaterials for light/heat conversion has motivated the development of a new research field, thermoplasmonics, ${ }^{12}$ which is impacting several areas: hot-electron chemistry, light harvesting, microfluidics and photothermal therapies. In particular, the heat sources used in photothermal therapy treatments are nanoparticles that emit heat upon excitation by electromagnetic radiation due to local plasmon resonance. ${ }^{1}$ Gold nanoparticles have attracted significant interest in biomedical applications due to their low toxicity and wide range of absorption wavelengths, targeting the so-called biological windows. ${ }^{4,13,14}$ Therefore, predicting the heat fluxes and temperature fields emerging from these hot particles is an important challenge. Addressing this challenge will allow significant advances in many areas, from medicine to materials processing. 
The ITC or its inverse, the Kapitza resistance, is crucial to quantify and explain heat transport across NP-fluid interfaces. The Kapitza thermal resistance arises from the different thermal transport properties of two phases in contact. ${ }^{15}$ The most vivid illustration of the Kapitza resistance is a significant temperature drop at subnanometer length-scales, namely at the interface between two materials or materials and fluids. Unfortunately, the ITC is often neglected in studies based on continuum theory since its experimental measurement is still scarce for nanoparticles and prone to significant uncertainties. ${ }^{16}$

Computer simulations provide a robust approach to compute the ITC by linking thermal transport to interatomic forces and relevant properties of solids and fluid phases, such as the thermal conductivity or the vibrational density of states. Molecular Dynamics (MD) simulation methods have evolved in different directions. Equilibrium methods (EMD) rely on the computation of cross-correlation functions, while Non-Equilibrium Molecular Dynamics (NEMD) mimics the experimental setup by explicitly simulating temperature gradients and heat fluxes across interfaces. Within NEMD, transient non-equilibrium MD (TNEMD) relies on fitting the time-dependent temperature relaxation to the solution of the heat diffusion equation. The fitting to a first-order decay law (the "lumped" method) gives direct access to the ITC. However, this approach neglects the thermal conductivity of the nanoparticle, a variable that is important at the nanoscale.

Models that include both the thermal conductivity and thermal conductance were proposed and applied to analyse the transient data of nanoparticles and biomolecules. ${ }^{17-20}$ One caveat of the TNEMD methods is that the heat diffusion equations are geometry dependent, making it challenging to analyse the heat transport of nanoparticles with contorted geometries. This issue prevents the identification of regions in the nanoparticle that might feature very different ITCs. Specifically, current TNEMD methods do not account for local thermal conductance in nanoparticles with complex geometries, such as facets, edges and vertices, and more generally, rough surfaces.

Stationary non-equilibrium MD (SNEMD) simulations provide another route to obtain 
the ITC, $G=J_{q} / \Delta T$, by computing explicitly the interfacial temperature jump, $\Delta T$, and interfacial heat fluxes, $J_{q}$. SNEMD has been applied to solid-liquid, ${ }^{21,22}$ liquid-vapour, ${ }^{23,24}$ and nanoparticle-fluid interfaces. ${ }^{16,25,26}$ Recently, the interplay of nanoparticle curvature and nanoparticle wettability was investigated, ${ }^{16}$ uncovering a correlation between high nanoscale curvature and high thermal conductance. This observation supports earlier studies of fluidfluid ${ }^{18}$ and nanoparticle-fluid interfaces ${ }^{27}$ as well as theoretical models. ${ }^{28}$ Recent SNEMD computations of gold nanoparticles in hexane, reported a dependence of the ITC on particle size too. However, the ITC increased with decreasing curvature. ${ }^{26}$ That work also reported the thermal conductance of faceted nanoparticles (cuboctahedral and icosahedral shapes), and proposed a correlation between the ITC and the local atomic environments in each nanoparticle via an atomic coordination number.

Previous SNEMD and TNEMD studies on NP thermal conductance relied on "bulkbinning" methods. These methods partition the simulation box into bins with simple, welldefined geometries, e.g., slabs or spheres, which are used to obtain density, temperature and heat flux profiles. This approach works well when considering structures with well-defined geometries, like spheres or planes. However, the bulk binning method averages out interfacial structural features that deviate from these geometries. By averaging the physical properties in each bin, the bulk binning approach assumes a continuous atomic distribution within a subvolume (bin) and isotropy in the interfacial properties of the regions sampled in a particular sub-volume. Real nanostructures feature roughness, facets and edges, where the heat flux and the temperature change rapidly depending on the specific location, hence introducing additional degrees of freedom that are difficult to model, e.g., at intersections between facets. Therefore, the heterogeneous structure of nanoparticle surfaces can potentially introduce markedly different local ITCs at subnanometer length scales. This situation is difficult to map into a simple geometry, hence resolving the ITC spatially, requires a higher level of computation to account for the heterogeneity of the interfaces.

We present in this work an algorithmic workflow, the Atomistic Nodal Approach (ANA), 
to quantify local thermal properties, namely the heat flux, temperature "jumps", and the ITC at angstrom level resolution. Our approach becomes particularly advantageous to investigate small NPs (few nm diameter) and anisotropic nanoparticles featuring under-coordinated atoms. Moreover, the ANA can be generalised to study a wide range of complex nanoparticle geometries without introducing specially designed binning methods to capture the nanoparticle shapes. We demonstrate the ANA method by investigating a wide range of nanoparticle

structures. We explain the dependence of the ITC of these nanoparticles in terms of the local coordination number of the surface atoms in contact with the surrounding fluid and identify the coexistence of high and low ITC regions in a nanoparticle. Finally, we discuss the general dependence of the ITC on nanoparticle size and geometry.

\section{Methods}

\subsection{Nanoparticle models and Simulation details}

We constructed nanoparticles of different geometries. The atoms in the nanoparticle and in the fluid interact through a Lennard-Jones potential,

$$
U(r)=4 \varepsilon\left[\left(\frac{\sigma}{r}\right)^{12}-\left(\frac{\sigma}{r}\right)^{6}\right]
$$

where $U$ is the pairwise interatomic interaction potential, $\sigma$ is the atomic diameter, and $\varepsilon$ is the interaction strength. Hereafter, we employ reduced units (denoted with an asterisk "*") to present our results. We define the interaction parameters as: $\sigma_{N P}^{*}=\sigma_{f}^{*}=1.0, \varepsilon_{f}^{*}=$ $1, \varepsilon_{n p}^{*}=10$, where $N P$ and $f$ refer to the nanoparticle and fluid, respectively. The cross interaction strength, $\varepsilon_{N P-f}$, was obtained using,

$$
\varepsilon_{\mathrm{NP}-\mathrm{f}}=F \sqrt{\varepsilon_{\mathrm{NP}} \varepsilon_{\mathrm{f}}}
$$

where $F$ is the heteroatomic interaction parameter set to 0.25 . This value of $F=0.25$ 
corresponds to a full wetting state, i.e., the NP-fluid contact angle is 0 degrees. ${ }^{16}$ The atomic interactions were truncated at $r^{*}=2.5$, and the linear and angular momentum of the nanoparticle were kept to zero at every step to maintain the position and orientation of the NP throughout the simulation. Previous studies of spherical nanoparticles employed the same LJ model, ${ }^{16}$ and the wettability of the nanoparticle was quantified as a function of $\varepsilon_{N P-f}$. Moreover, the LJ model has been parametrized to investigate fcc cubic metals, showing excellent performance in terms of densities, surface tensions, metal-water interfacial properties, and mechanical properties. ${ }^{29}$ Therefore, this generic model represents a good starting point for our investigation and to test our numerical approach.

Our simulations involved several steps. First, we assigned random velocities to the particles, drawn from the Maxwell-Boltzmann distribution at $T^{*}=0.90$. Afterwards, we equilibrated the system using the Nosé-Hoover thermostat at $T^{*}=0.90$ with a relaxation time of $0.5 \tau^{*}$. Nanoparticle heating can be achieved in an experimental setting using optical or magnetic excitation. Following the equilibration period, we simulated the heating of the nanoparticle using a simple velocity rescaling thermostat applied to the core of the nanoparticle. The thermostatting volume was defined at a radial distance $d_{R h o t}^{*}=2$ within the NP surface identified using the $\alpha$-shape algorithm. We set a cold thermostat at a radial distance $d_{R_{\text {cold }}}^{*}=7$ away from the NP surface. The hot and cold regions generate a constant heat flux in the stationary state.

For all simulations reported in this work, the lattice parameter of the NP prior to equilibration was set to $l_{\mathrm{c}}=\left(4 / \rho_{\mathrm{NP}}\right)^{1 / 3}$, with the NP density set to $\rho_{\mathrm{NP}}^{*}=\rho_{\mathrm{NP}} \sigma^{3}=1$. The temperature $T$ of each particle of mass $m_{i}$ and velocity $v_{i}$ in the simulation was determined using the equipartition equation:

$$
T=\sum_{i=1}^{N} \frac{m_{i} v_{i}^{2}}{k_{B} N_{d o f}},
$$

where $k_{B}$ is the Boltzmann constant and $N_{\text {dof }}=3$ is the number of translational degrees of 
freedom for each particle, i.e. the dimensionality of the system. To determine the heat flux at each position, we used a local version of the formula derived by Irving and Kirkwood: ${ }^{30}$

$$
\boldsymbol{J}_{q, i}=\frac{1}{V_{i}}\left[\frac{1}{2} m_{i} \boldsymbol{v}_{i}^{2} \boldsymbol{v}_{i}+\phi_{i} \boldsymbol{v}_{i}+\frac{1}{2} \sum_{j \neq i}\left(\boldsymbol{v}_{i} \cdot \boldsymbol{F}_{i j}\right) \boldsymbol{r}_{i j}\right]
$$

where $\boldsymbol{J}_{q, i}$ is the heat flux corresponding to particle $i$ with associated volume $V_{i}$, which we calculate using the Voronoi polyhedra approach (see below). The first term in equation (4) represents the kinetic energy flux of particle $i$ and the second term its potential energy flux, where $\phi_{i}$ is the potential energy of particle $i$ in the field of all the other particles. The third term, referred to as the collisional contribution, takes into account the flux contribution to the forces between particle $i$ and $j$ separated by a distance $r_{i j}$, and it is the dominant contribution in dense fluids and solids.

A similar equation can be written for a control volume (V) used in the "bulk binning" method,

$$
\boldsymbol{J}_{q}(V)=\frac{1}{V}\left[\sum_{i=1}^{N \in V}\left(\frac{1}{2} m_{i} \boldsymbol{v}_{i}^{2} \boldsymbol{v}_{i}+\phi_{i} \boldsymbol{v}_{i}\right)+\frac{1}{2} \sum_{j \neq i}\left(\boldsymbol{v}_{i} \cdot \boldsymbol{F}_{i j}\right) \boldsymbol{r}_{i j}\right]
$$

The specific version of the Irving Kirkwood derivation in Eq. (4) is used to obtain the local heat flux on each atom in the nanoparticle. The location of the NP atoms is well defined throughout the simulation (with only minor vibrations about their equilibrium positions), i.e. a given atom does not diffuse from its initial position, and it occupies the same relative position with respect to the other NP atoms throughout the simulation. This allows us to calculate the per-atom local volume, $V_{i}$, which we identify with the Voronoi volume that is calculated for each atom as a function of time. Practically, the per-atom volume, $V_{i}$, was obtained using the Voronoi construction of both fluid and nanoparticle atoms in SNEMD simulations spanning $5 \times 10^{4}$ steps and ten independent runs (see Figure $7(\mathrm{~b})$ for a visualisation of a Voronoi tessellation).

The simulation workflow was divided into three stages: initialisation for $10^{6}$ steps and 
NVT equilibration under Nosé-Hoover thermostat, followed by applying the velocity rescaling thermostat at every timestep at the hot and cold regions to generate stationary states using SNEMD. The initial $5 \times 10^{4}$ time-steps were discarded, and the remaining $5 \times 10^{4}$ production steps were used for heat transfer measurements. The resulting trajectories were then used to perform the analyses either with the bulk binning or the ANA approaches. Throughout the simulations, the angular and linear momentum of the NP is reset to zero every step to keep the NP stationary. The trajectories were generated using LAMMPS (19Mar2020 version), ${ }^{31,32}$ with a time-step $0.0025 \tau^{*}$.

\section{$2.2 \quad$ Bulk Binning Approach}

Previous computational studies of the ITC $^{16,19,26}$ have predominantly employed the bulk binning approach (BBA), where the heat flux and temperature profiles are obtained from averaging the thermal properties of all atoms inside sampling volumes located between the thermostats and in the direction normal to the interface of interest (see Figure $1 \mathrm{a}, \mathrm{b}$ ). We performed BBA calculations for specific particle geometries to provide a benchmark for our implementation of the ANA method. Specifically, we computed density, temperature and heat flux profiles, using trajectories spanning $5 \times 10^{4}$ steps. We performed five independent runs to obtain the final averages and calculate statistical uncertainties. The ITC was obtained from the analysis of the temperature and heat flux profiles by fitting them to the continuity equations. This is further elaborated in the SI (section 2). 

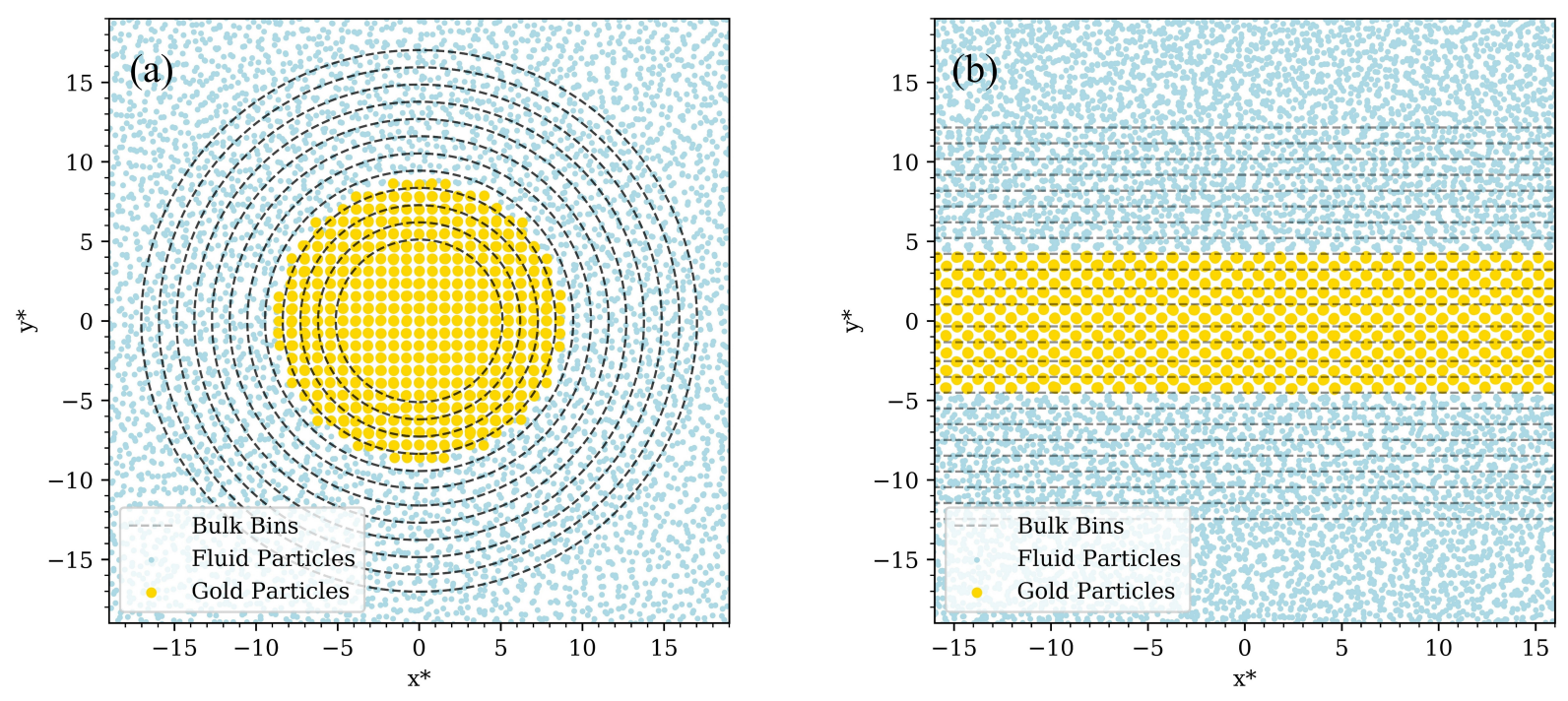

Figure 1: Illustration of BBA for (a) spherical or cylindrical (top view) shells and (b) slabs for flat surfaces. These methods use a smooth and continuous shell to approximate the surface properies.

We used the BBA route to quantify the ITC of nanocylinders and nanospheres (see Figures 5 (a), (c)). The cylindrical nanoparticle spanned the whole simulation box in the axial direction; hence we considered only the radial heat flux since there is no temperature gradient nor heat flux in the axial direction.

\subsection{The Atomistic Nodal Approach}

\subsubsection{The philosophy behind ANA}

Unlike the bulk binning approach, the ANA makes no assumptions about the NP geometry. Instead, it projects local temperatures and heat fluxes on each atom ("node") in the NP. In addition, it uses various computational geometry techniques to identify the atoms defining the interface and the ITC.

A cuboid grid is used to calculate the local properties of the fluid atoms around the NP. The calculations are performed on a per-atom and a per-grid basis for NP and fluid atoms, respectively. We show in figure 2 an example of a nanoparticle immersed in a fluid, which illustrates the definitions used for the NP nodes and fluid grids used in this work. 

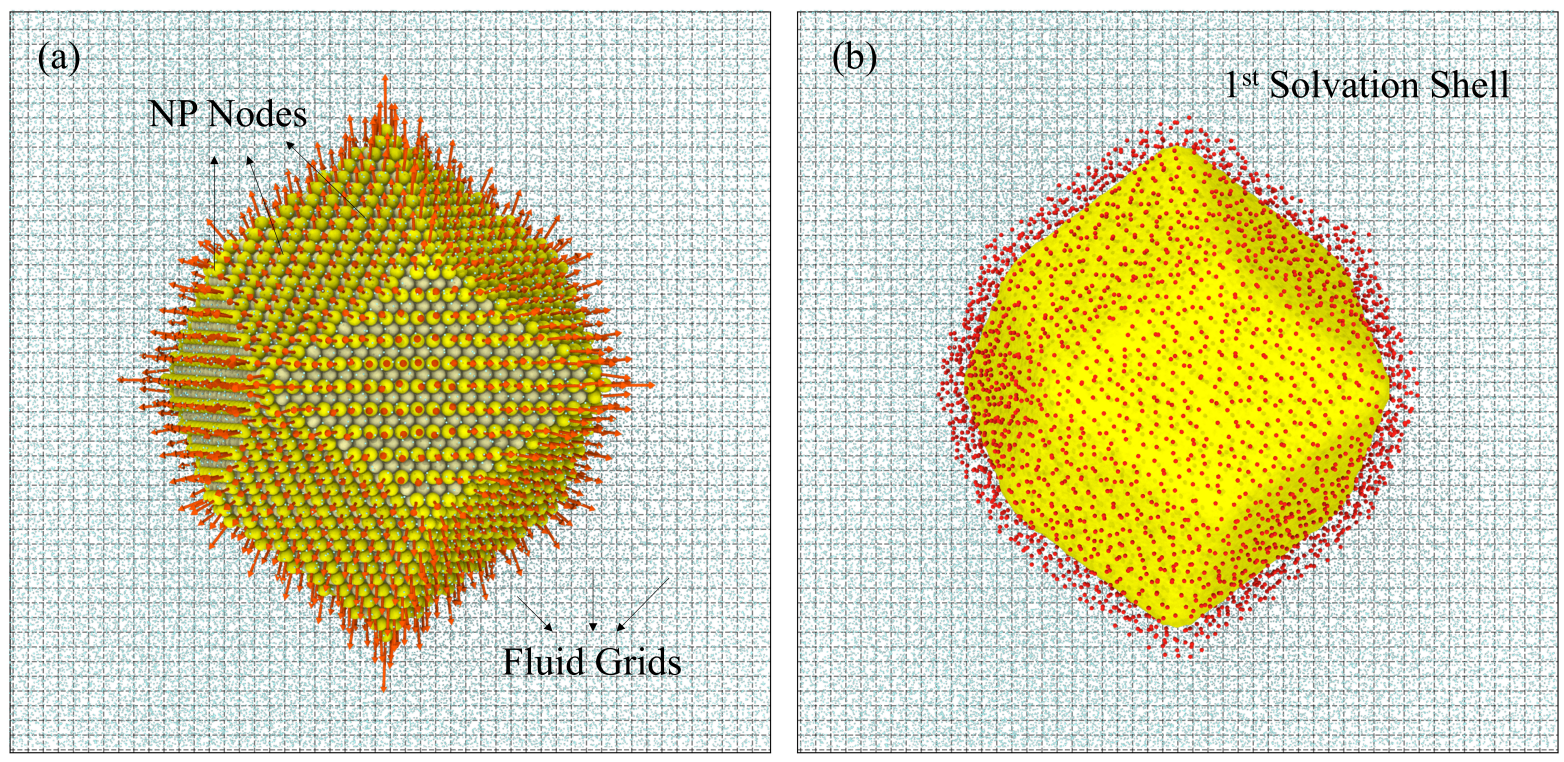

Figure 2: (a) Illustration of the fluid (blue) and NP atoms (yellow), and the heat flux vectors (red arrows) for a rhombic-dodecahedron gold nanoparticle. The NP atoms are treated as nodes. A cubic grid of length $0.4 \sigma$ is superimposed on the fluid region to compute the properties of the fluid atoms. (b) The surface (yellow) of the NP identified by the $\alpha$-shape algorithm. The atoms in the nanoparticle solvation shell are highlighted in red.

Following the calculation of the averaged per-atom data from the NP coordinates, we employed the $\alpha$-shape algorithm, ${ }^{33,34}$ which was used to construct a triangular surface around the NP's, using a spherical probe with $\alpha$-radius $R_{\alpha}^{*}=1.1$, which corresponds to the distance of the first maximum of the NP-NP radial distribution function (see Figure S13). The probe radius is an important variable in our method. Our choice $\left(R_{\alpha}^{*}=1.1\right)$ corresponds to the nearest neighbour distance between atoms belonging to the nanoparticle. This probe radius identifies under-coordinated atoms at the nanoparticle surface and prevents the inclusion of atoms that do not belong to the surface in the ITC analysis.

Subsequently, the temperature gradient $\nabla \mathrm{T}(x, y, z)=\left(\frac{\partial T}{\partial x}, \frac{\partial T}{\partial y}, \frac{\partial T}{\partial z}\right)$ was calculated in order to determine the direction of heat flow at the NP-fluid interface. $\nabla \mathrm{T}(x, y, z)$ was computed using tri-linear interpolation methods, further elaborated in the SI (see section $3)$. The direction of the vector $\nabla \mathrm{T}(x, y, z)$ was observed to be largely parallel to the normal of flat faceted surfaces. We employ in all our computations of the ITC the heat flux in the direction of the thermal gradient. 
To calculate the interfacial temperature "jump", $\Delta T$, i.e., the NP-fluid temperature discontinuity per atom, we used the temperature of the fluid grids within the first solvation shell of the NP's surface atoms. To find the nearest grids, we constructed a binary tree for both the fluid and particle atoms. The resulting k-dimensional tree (KD-Tree) was built by dividing the space occupied by the fluid grid into cells using the 'sliding midpoint' method. ${ }^{35}$ Each node on the tree represents a partition of the space surrounding a reference point (i.e., a surface NP atom) that allows to quickly query the fluid (F) nearest neighbours $\mathcal{N}_{N P}$ whose radii $\mathbf{r}_{N P}-\mathbf{r}_{F}$ are within a fixed distance $M_{1}$ of the atom in the NP, where $M_{1}$ is the first minimum in the NP-fluid radial distribution function (Figure S13a),

$$
\mathcal{N}_{N P}=F:\left|\mathbf{r}_{N P}-\mathbf{r}_{F}\right|<M_{1}
$$

After identifying the nearest neighbours for each atom at the surface of the NP, we computed the local temperature, density and temperature-gradient $\nabla \mathrm{T}$. We used a density weighted average across all local neighbours to compute the fluid temperature in each grid box in Figure 2. The density weighted average was used to ensure that each neighbouring grid property is determined by the fluid density $\rho_{F}$ of each grid, to avoid over-sampling underpopulated regions. The temperature discontinuity $\Delta T$ corresponding to each NP atom, $i$, at the surface is calculated from:

$$
\Delta T_{i}=T_{N P, i}-\frac{\sum_{F \in \mathcal{N}_{N P, i}} \rho_{F} T_{F}}{\sum_{F \in \mathcal{N}_{N P, i}} \rho_{F}}
$$

where $T_{N P, i}$ and $T_{F}$ are the per-atom NP (atomic node, $i$ ) and fluid grid temperatures, respectively. 


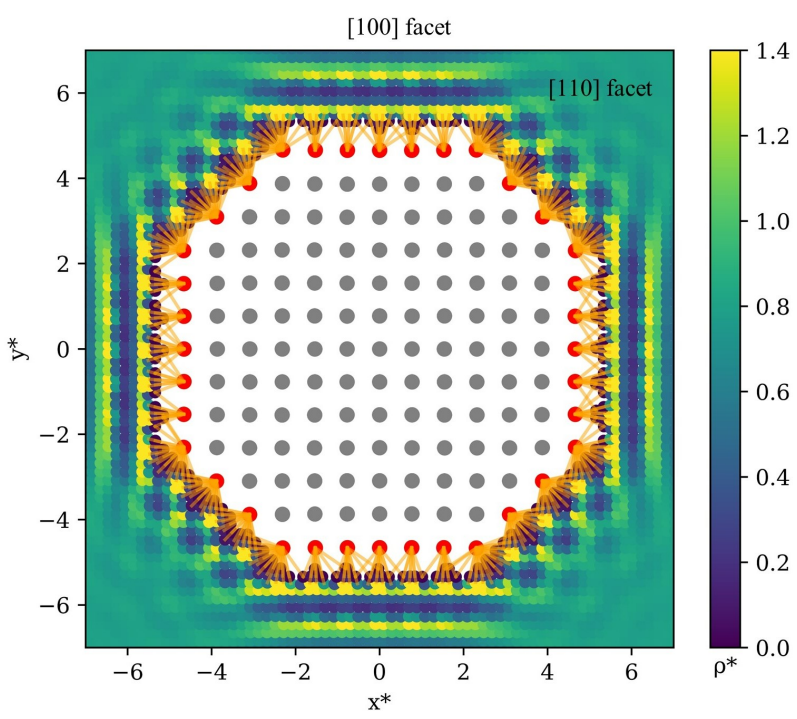

Figure 3: Density profile of the fluid surrounding the NP. The high density fluid regions are represented in yellow, and the low density regions in purple. The NP surface-atoms are coloured in red, and the rest of the NP atoms are in grey. Orange lines joining NP surface atom with fluid atoms indicate the nearest fluid-neighbours $\mathcal{N}_{\mathcal{N} \mathcal{P}}$ identified.

The ITC was obtained by using the heat flux, $J_{q}$, projected along the temperature gradient, and the corresponding $\Delta T$ (from Eq. (7)) obtained for each NP surface-atom,

$$
G=\frac{\mathrm{J}_{\mathrm{q}}}{\Delta T}
$$

We note that the $\alpha$-shape sorting method discussed above gives access to other important local properties, such as the coordination number between atoms in the nanoparticle $(\mathrm{CN})$, and the number of fluid atoms solvating a given atom $i$ located at the surface of the nanoparticle. which can be extracted from $\mathcal{N}_{N P, i}$.

\subsubsection{General ANA Simulation approach}

Figure 4 shows the flow diagram employed in the ANA introduced in this work. Firstly, we identify the core atoms and apply the hot thermostat on those atoms up to $r^{*}=2$ away from the nanoparticle surface defined by the $\alpha$-shape algorithm. The calculations are performed using "computational farming", namely, we run $10^{3}$ simultaneous independent simulations, 
$5 \times 10^{4}$ steps each, and we use these simulations to obtain averages. We then calculate the local heat flux using the IK Eq. (4) and the local temperature gradients using linear interpolation (see section 3 in the SI). The projected heat flux along the direction of thermal gradient and the local $\Delta T$ (Eq. (7)) give the ITC.

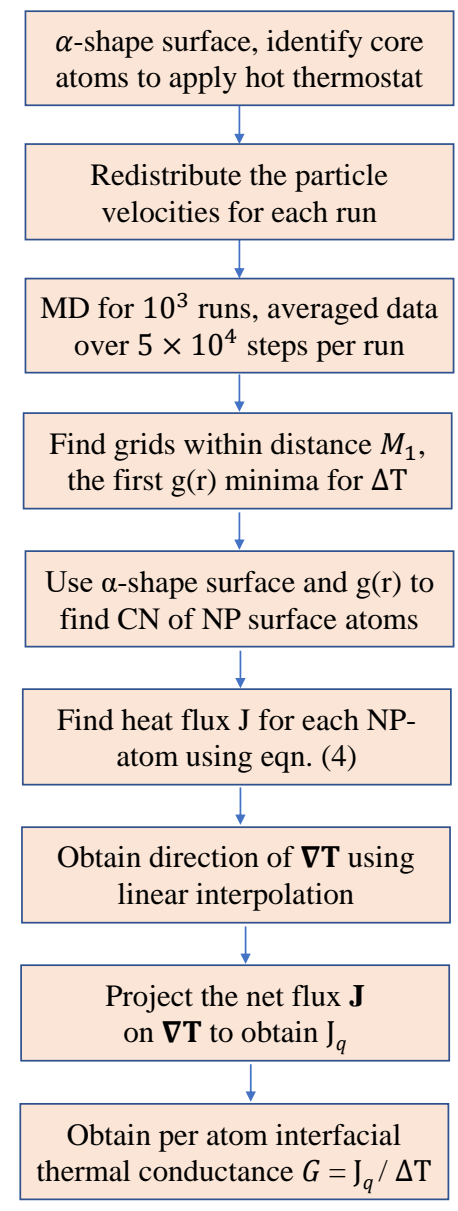

Figure 4: Algorithmic approach used to analyse the NEMD data generated with the ANA method. The ANA method makes use of the computational geometry methods, $\alpha$-shapes to identify surface atoms, and kd-trees to find nearest neighbours for the NP surface-atom nodes.

Except for long rectangular cuboids used for the octagonal nanowire specified later, all other simulations used cubic grids of $l^{*}=0.4$. This size was optimized to obtain the local fluid properties at a good resolution. For each NP atom, a unique identification number was assigned, which allowed the averaging of their thermodynamic properties across different simulations. Information on the particle dimensions and simulation box dimensions are 
provided in the SI, section 1.

\section{Results and Discussion}

\subsection{Comparison between BBA and ANA approaches}

We show in Figure 5 the temperature and heat flux profiles of cylindrical and spherical nanoparticles obtained with the ANA and BBA approaches. For the BBA calculations we used cylindrical binning for nanowires, and spherical binning for nanospheres. The temperature profiles obtained with both methods agree within the error of our computations. The temperature profile of nanowires in the bulk region follows the logarithmic decay predicted by the heat diffusion equation (see SI for a derivation of this equation),

$$
T_{p}(r)=-\frac{q_{r}}{2 \pi \kappa_{p} L} \ln \left(\frac{r}{r_{c}}\right)+T_{c}^{P}
$$

where $T_{p}(r)$ is the nanoparticle temperature at radial distance $r, L$ the nanowire length, $\mathrm{q}_{\mathrm{r}}=|\Delta U / \delta t|$ is heat rate, obtained from the average amount of energy exchanged at the hot or cold thermostats in time $\delta t, \kappa_{p}$ is the thermal conductivity of the NP, $r_{c}$ the cylindrical radius at the NP-Fluid boundary and $T_{c}^{P}$ the temperature of the NP at $r_{c}$. For the sphere, we recover an inverse relationship between the NP and fluid temperature with radial distance $R$ as described in ref. ${ }^{16}$ (see SI for derivation). We fitted the temperature radial profiles (from the region between the hot and cold thermostat) between the thermostats to the theoretical equations using an error function to model the temperature discontinuity at the interface:

$$
T(r)=\frac{1}{2}\left[T_{p}(r)+T_{F}(r)-\left(T_{p}(r)-T_{F}(r)\right) \operatorname{erf}\left(\frac{\sqrt{\pi}\left(r-r_{c}\right)}{\omega}\right)\right],
$$

where $T_{F}(r)$ is the fluid temperature that follows a similar form to eq. (9) and $\omega$ quantifies the

steepness of the error function $\operatorname{erf}\left(\frac{\sqrt{\pi}\left(r-r_{c}\right)}{\omega}\right) \cdot{ }^{36}$ The values of $\kappa$ obtained from the fitting to eqn.(9) match those reported by Tascini et. al. ${ }^{16}$ Eq. (10) was used to fit all the temperature 
profiles investigated in this work, and to calculate the temperature "jump", $\Delta T$.
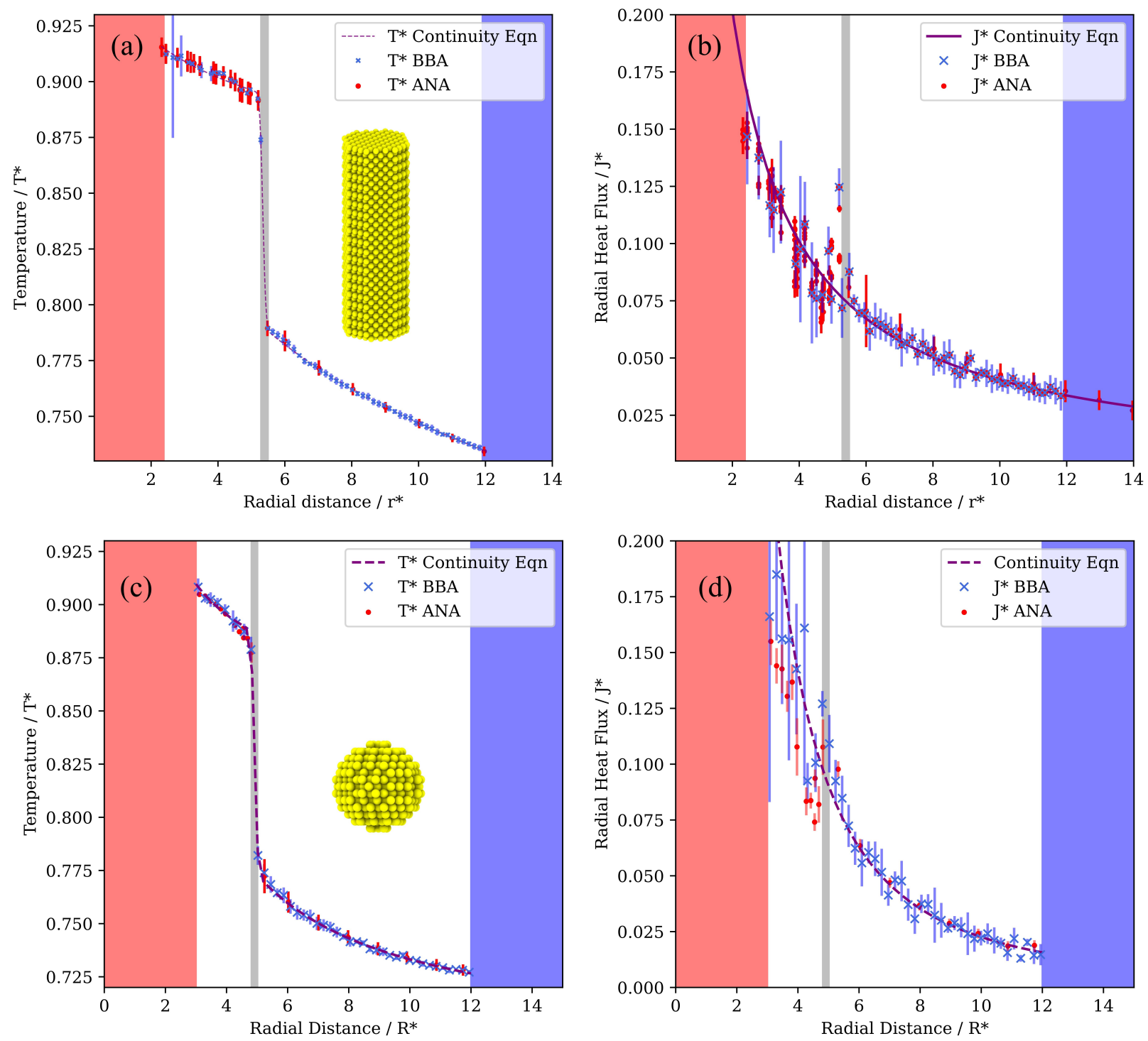

Figure 5: Dependence of the temperature (panels (a) and (c)) and heat flux (panels (b) and (d)) profiles with radial distance for cylindrical and spherical nanoparticles obtained with the ANA and BBA methods. The red and blue areas represent the location of the hot and cold thermostats, respectively. The temperature profiles and the heat fluxes obtained with the BBA and ANA approaches agree with the profiles predicted by equation (9) for the fluid and the nanoparticle. We show in the insets of panels (a) and (c) a cylindrical NP of radius $r^{*}=5.6$, and a spherical NP of radius $R^{*}=5.0$.

We show in Figure 5 the temperature profiles and radial heat fluxes around cylindrical and spherical nanoparticles. The results obtained with the BBA and ANA methods agree within the statistical uncertainty of our computations, showing that our implementation of 
the local heat flux (see Eq. (4)) produces consistent results with the heat fluxes obtained using a cylindrical or spherical sampling volume (via Eq. (5)). The radial heat fluxes agree with the results of the continuity equations (see Figures $5(\mathrm{~b})$ and $(\mathrm{d})$ ) continuity equation, $\mathrm{J}_{\mathrm{q}}=\mathrm{q}_{\mathrm{r}} /(2 \pi L r)$ for cylinders and $\mathrm{J}_{\mathrm{q}}=\mathrm{q}_{\mathrm{r}} /\left(4 \pi R^{2}\right)$ for spheres. The continuity result provides an additional consistency check for the heat flux computations using the IK method (Eqs. (4) and (5)). We find excellent agreement between the continuity equation and both IK methods), supporting the validity of our ANA implementation. Eqn. (10) accounts accurately for the temperature profiles, too (see Figures Figure 5(a) and (c)), reproducing the local temperature inside the nanoparticle, fluid and interface.

\subsection{Relationship between Coordination Number and Conductance: The spherical nanoparticle}

We have shown above that the ANA method produces temperature profiles and heat fluxes consistent with the calculations using common control volumes and the continuity equations. In the following, we use the ANA approach to obtain the local ITC of nanoparticle-fluid interfaces. We will establish a relationship between the ITC and the local structure of the NP. With this purpose, we use the local coordination number between nanoparticle atoms $(\mathrm{CN})$ as a fingerprint characterizing their local environment. In this context, "low" values of $\mathrm{CN}$ indicate a more significant number of contacts between that NP atom and the fluid atoms. We note that even for shapes of high symmetry, like spheres, the nanoscale roughness of the surface results in unique atomic environments, and therefore somewhat different local coordination numbers. We illustrate this notion in Figure 6, for a sphere of diameter $2 R^{*}=40(\sim 12 \mathrm{~nm}$ using $\sigma=0.3 \mathrm{~nm}$, which is of the order of the water molecule diameter). The surface of this spherical nanoparticle features a heterogeneous environment with the coordination numbers of the surface atoms spanning values between 6 and 9 . Undercoordinated atoms, with $\mathrm{CN}$ values lower than bulk $\left(\mathrm{CN}_{b u l k-N P}=12\right)$, feature higher ITCs. This result is consistent with a larger number of contacts with the fluid, which reduces 
the thermal resistance. Indeed, NP surface-atoms with low CN, feature higher local fluid densities (see Figure S13).
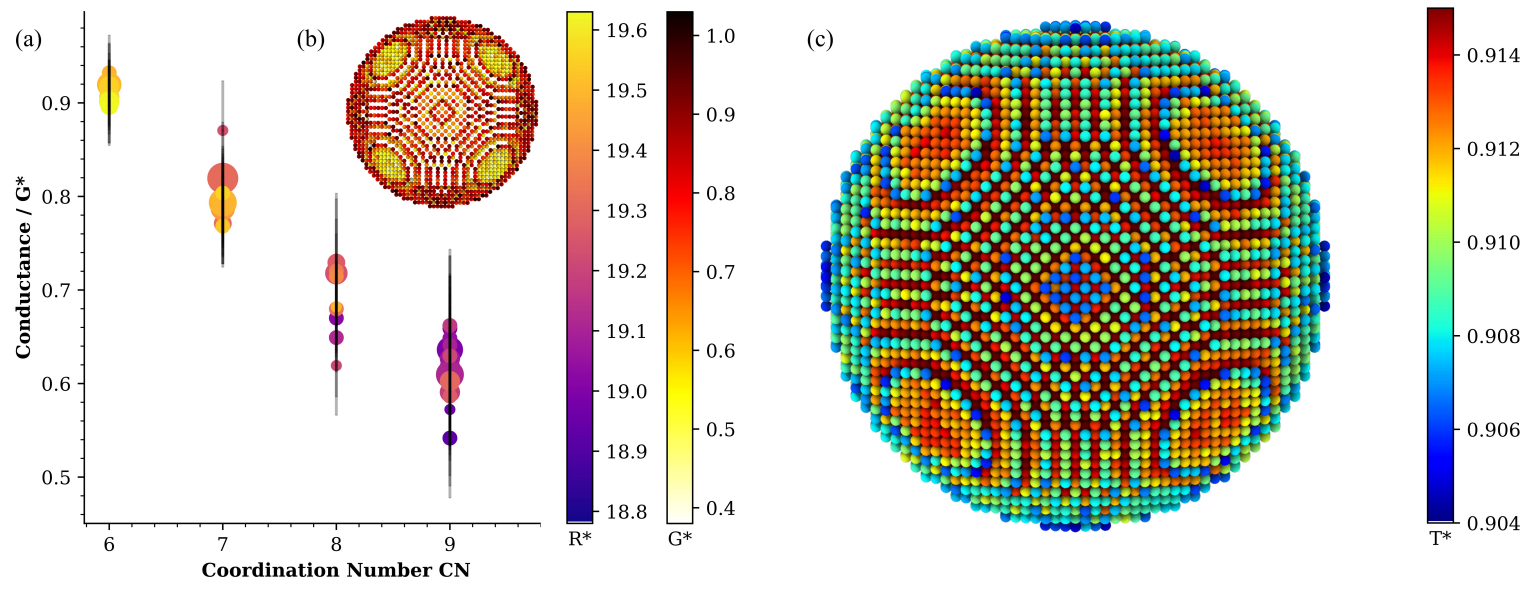

Figure 6: (a) Dependence of the ITC with the local CN and radial distance for a spherical nanoparticle of $R^{*}=20$. The color maps indicate different thermal conductances, $G^{*}$, or radial distances, $R^{*}$. (b) The ITC results have been projected on the NP surface-atoms to identify regions of high and low conductance. (c) Temperature distribution of the NP atoms, with hotter regions corresponding to atoms with low thermal conductance and vice-versa. The size of the points in panel (a) indicates the ratio of surface atoms with a particular CN and radial distance $R^{*}$ from the NP center.

Figure 6(b) shows a color map of the ITC projected on the NP surface atoms. Our ANA method uncovers regions with contrasting thermal conductance. Facets with high $\mathrm{CN}=9$ feature significantly lower ITCs than regions with low $\mathrm{CN}=6$. The differences in conductance between these regions, are very significant, $\sim 50 \%$. The surface atoms with lower conductance experience a larger temperature "jump", and are hotter than the high conducting ones. Atoms with high ITC dissipate heat better and cool down faster. Conversely, the fluid surrounding low ITC regions remains cooler due to poorer heat transfer (see Figure 6(b) and (c)). Overall, the ANA approach uncovers important details missed by previous methods, such as bulk binning, which only provides an ITC corresponding to the average over regions with very different ITCs. We have compared the thermal conductance obtained from both methods, ANA and BB, by computing the arithmetic mean of the local ITCs obtained with 
the ANA approach. We obtain $G^{*}=0.74 \pm 0.02$, in good agreement with the result from the radial bulk binning method, $G^{*}=0.75 \pm 0.05$, showing the consistency of the ANA method.

\subsection{ITC of complex geometries: Induced Edge Effects on Conduc-}

\section{tance}

We have shown that the ANA allows for calculating local ITCs in relatively simple geometries such as spheres. In the following, we use the ANA method to compute the ITC of complex nanoparticles and resolve the different contributions due to atoms located at facets, edges and vertices.

Firstly, we investigated an infinite nanowire with an octagonal cross-section and an average cylindrical diameter $2 R^{*}=11.1(3.3 \mathrm{~nm}$ using $\sigma=0.3 \mathrm{~nm})$. This NP consists of undercoordinated atoms at edges and high CN atoms at the faces (100) and (110). We show in Figure 7 the NP structure along with local ITC maps projected onto the NP surfaceatoms. We have represented our results as a function of the radial distance to the main axis of the NP. Note that atoms belonging to the same facet in this representation might have a different radial distance to the axis. The ITC varies significantly depending on the atom location, and the ITC decrease in the order: edges $>(110)$ facets $>(100)$ facets. Following the discussion in the previous section, we can explain the decrease of the ITC in terms of the local $\mathrm{CN}$ of the surface atoms. The $\mathrm{CN}$ increases in the order 6,7 for edges, (110) and 8 for (100) facets, respectively, and we get consistently higher ITC for (110) faces. This result supports our view that lower CN is connected to higher ITC.

The ITC of the NP atoms located at (110) and (100) faces are very similar to those obtained simulating periodically extended surfaces with that symmetry (see Figure 7(a)). This result indicates that the ITC of the nanoscale facets can be approximated with the ITCs of macroscopic surfaces. We also calculated the average ITC of the nanoparticle by using the arithmetic mean of the ITCs and considering the fraction $(f)$ of surface atoms with different CN: $8(f=20 / 36), 7(f=8 / 36), 6(f=8 / 36))$. Despite the smaller fraction of 
atoms with lower CN, they contributed significantly to the average, due to their high ITC. We obtained $G^{*}=0.724 \pm 0.005$ using the arithmetic mean, which is in agreement with the coarse-grained radial bulk-binning method, $G^{*}=0.74 \pm 0.01$.

It is worth noting the slight increase of the ITC from the center of (100) facet (lower radial distance) towards the edges (higher radial distance) (see Figure 7a). All the atoms in this facet have the same CN (8). These small changes in ITC are therefore not associated with different coordination numbers. The Voronoi volume analysis helps rationalize the observed increase in conductance near the edges, as it considers the packing effects of both fluid and NP atoms surrounding each surface atom. Therefore, it can identify subtle variations in the local atomic environment.

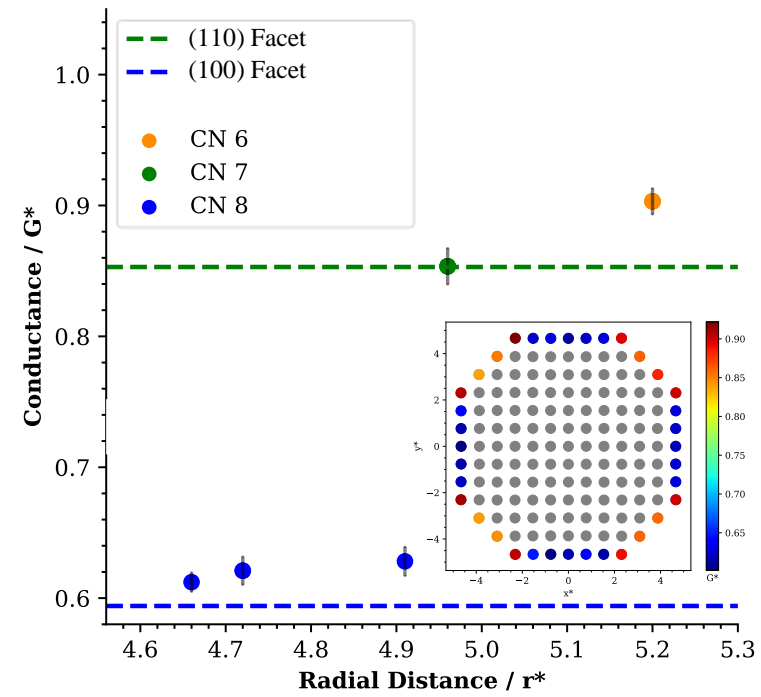

(a)

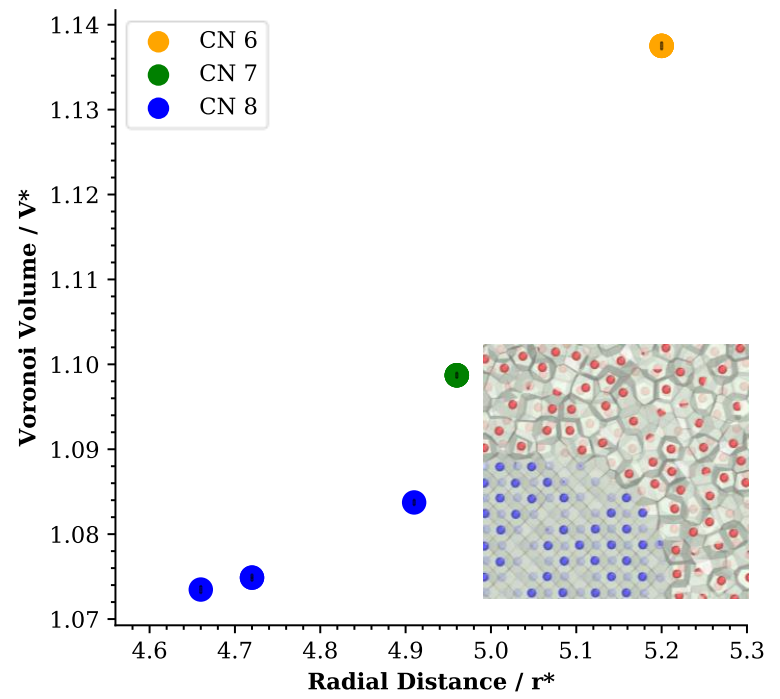

(b)

Figure 7: (a) Dependence of the ITC with the radial distance of the atoms to the main axis of nanowire periodic in the axial direction (infinite length). The dashed horizontal lines represent the ITC obtained with independent simulations of periodic (infinite) surfaces spanning the whole simulation cell. The inset shows a cross section of the nanoparticle. The heat map indicates the ITC of the atoms at the surface of the NP. The bulk atoms in the NP are shown as grey spheres. (b) Voronoi volumes as a function of the radial distance. The insert features the fluid atoms (red) and NP atoms (blue) and their corresponding Voronoi tessellation.

The Voronoi volume per atom increases as the atoms in the (100) facet approach the edges 
(larger radial distance) (see Figure 7b), hence providing a correlation with the slightly higher conductance reported in Figure 7a. Consistently, the atoms in the (110) face $(\mathrm{CN}=7)$ and the edges $(\mathrm{CN}=8)$, which have higher ITC, do also feature higher Voronoi volumes. The Voronoi analysis is useful, since it shows that atoms in the same facet with with the same CN can have slightly different volumes, which ultimately indicates whether the atoms are in the core of the facet (lower volumes) or towards the edges (higher volumes). A higher atomic Voronoi volume is also observed to correlate with greater NP-fluid interactions. Since the fluid has lower density than the NP, and surface NP atoms that are uncoordinated with other NP atoms bind more with the less dense fluid, the Voronoi volume of the undercoordinated NP atoms is increased.

\subsection{Thermal conductance of complex nanoparticle shapes}

Nanoparticles can be synthesized in various complex shapes incorporating in their structure different facets and surface atoms with different coordination numbers. We analyse in the following the ITC of different shapes corresponding to experimental structures. Some of these shapes, e.g. cylinders or particles featuring triangular faces, i.e., with "spikes", can potentially be of interest in thermal therapy applications. ${ }^{37}$

We correlate the ITC of the different NP with the average coordination number, $\mathrm{CN}_{\mathrm{avg}}$. Our calculation relies on the $\alpha$-shape method to identify surface atoms. For the latter, we consider only atoms whose coordination number is $\leq 9$. We provide a justification for this criterion in the SI figure $\mathrm{S} 15$. We define the $\mathrm{CN}_{\mathrm{avg}}$ of a particle as,

$$
\mathrm{CN}_{\mathrm{avg}}=\frac{\sum_{i=1}^{9} i \cdot s_{i}}{\sum_{i=1}^{9} s_{i}}
$$

where the index $i$ runs over coordination numbers $1 \cdots 9$, and $s_{i}$ is the number of atoms with coordination number $i$. The number of surface atoms is proportional to the surface area of the NP. 
To calculate $\mathrm{CN}_{\text {avg }}(\mathrm{n})$ we use the analytical equations derived by Kaatz et al. ${ }^{38}$ for archimedean and platonic solids whose size and number of atoms are linked to the number of complete shells, $n$, that it is composed of (see ref. ${ }^{38}$ for further details). The analytical solutions from Kaatz et al.'s equation are collected in Figure 8, along with a representation of the NP shapes and examples of these shapes taken from experimental studies. The $\mathrm{CN}_{\mathrm{avg}}(\mathrm{n})$ of various platonic and archimedean solids can be defined in terms of a rational expression consisting of a second-order polynomial in the number of surface shells, such that $\mathrm{CN}_{\mathrm{avg}}(\mathrm{n})=\frac{\sum_{i=1}^{9} i s_{i}(n)}{\sum_{i=1}^{9} s_{i}(n)}$. Using $\mathrm{CN}_{\mathrm{avg}}(\mathrm{n})$ and the equations provided in reference ${ }^{38}$ we get the rational expression:

$$
\mathrm{CN}_{\mathrm{avg}}(\mathrm{n})=\frac{a n^{2}+b n+c}{d n^{2}+e n+1}
$$

where $a \cdots e$ are coefficients of second order polynomials listed in figure 8 . The denominator in equation (12) quantifies the total number of surface atoms with $C N \leq 9$. The ratio of coefficients $a / d$ quantifies the highest surface coordination environment attainable for a particle in the limit $n \rightarrow \infty$. Shapes with $\lim _{n \rightarrow \infty} C N_{\text {avg }}(n)=9.0$ (tetrahedron, octahedron, dodecahedron and decahedron) consists of (111) facets, only.

Non-archimedean and non-platonic shapes like spheres and cylinders do not possess such magic numbers. Hence, the numerator of Eq. (11), $\sum_{i=1}^{9} i s_{i}$, is a second order polynomial in $r$ for spheres $\left(4 \pi r^{2}\right)$, and first order for cylinders of length $L,(2 \pi r L)$, where $r$ is the NP spherical and cylindrical radii respectively. The resulting equation, $\mathrm{CN}_{\mathrm{avg}}(\mathrm{r})=\frac{\mathrm{ar}^{2}+\mathrm{br}+\mathrm{c}}{\mathrm{dr} \mathrm{r}^{2}+\mathrm{er}+1}$, resembles eqn. (12) where $a \cdots e$ are coefficients obtained by fitting the dependence of the average surface coordination number with the number of atoms in the nanoparticle (see figure 9).

$\mathrm{CN}_{\text {avg }}(\mathrm{n})$ increases in a similar fashion with the number of atoms for nanoparticles of different shapes (see Figure 8). Nanoparticles with $8.0<\lim _{n \rightarrow \infty} C N_{\text {avg }}(n)<9.0$ (cuboctahedron and truncated octahedron) have both (100) and (111) faces. The ratio of the surface area of these faceted surfaces determines $\lim _{n \rightarrow \infty} C N_{\text {avg }}(n)$. The hexagonal bipyramid NPs consist of (111) and (210) facets, resulting in a $\lim _{n \rightarrow \infty} C N_{\text {avg }}(n)=8.5$, 
an average of coordination numbers 9 and 8. Cubes are constructed using (100), hence $\lim _{n \rightarrow \infty} C N_{\text {avg }}(n)=8.0$. Lastly, the shape of the rhombic dodecahedron is dominated by (110) resulting in $\lim _{n \rightarrow \infty} C N_{\text {avg }}(n)=7$.

We show in Figure 9 the dependence of the average coordination number of the surface atoms for different shapes taking into account the NP size. The $\mathrm{CN}_{\text {avg }}(\mathrm{n})$ increases with the number of atoms in the NP. This increase is monotonic, except for some shapes (see Figure S17 in the SI). Following the results from the previous section, we therefore expect that the ITC will decrease as the particle size increases. We also find that the rhombic dodecahedron features the lowest $\mathrm{CN}_{\text {avg }}(\mathrm{n})$, while icosahedrons, decahedrons, tetrahedrons and octahedrons possess the highest $\mathrm{CN}_{\mathrm{avg}}(\mathrm{n})$. Advancing the discussion below and considering our analysis in the previous section, we expect that these structures will show the lowest (icosahedron) or highest (rhombic dodecahedron) ITCs. We conclude from this analysis that amongst the various NP types investigated, spherical, cylindrical and rhombic dodecahedron shapes are likely to have higher conductances. 
(a) Tetrahedron
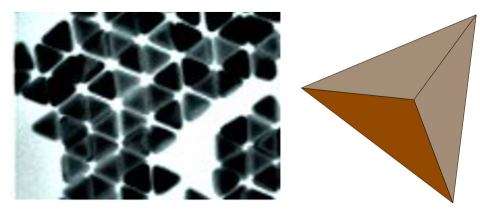

$\mathrm{CN}_{\text {avg }}(\mathrm{n})=\frac{3\left(3 n^{2}-3 n+2\right)}{n^{2}+1}$

$\lim _{n \rightarrow \infty} C N_{\text {avg }}(n)=9.0$

(d) Octahedron
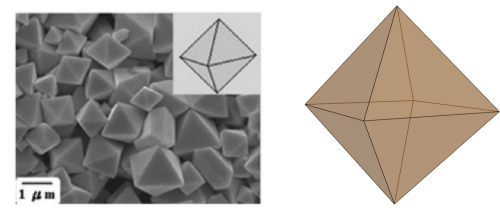

$$
\begin{gathered}
\mathrm{CN}_{\mathrm{avg}}(\mathrm{n})=\frac{6\left(3 n^{2}-2 n+1\right)}{2 n^{2}+1} \\
\lim _{n \rightarrow \infty} C N_{\mathrm{avg}}(n)=9.0
\end{gathered}
$$

(g) Icosahedron
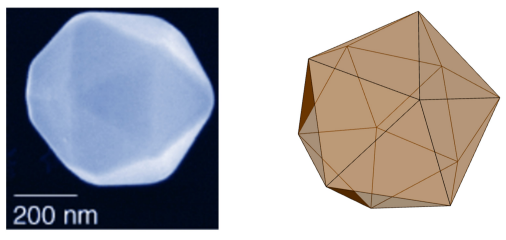

$$
\begin{gathered}
\mathrm{CN}_{\mathrm{avg}}(\mathrm{n})=\frac{3\left(15 n^{2}-5 n+2\right)}{5 n^{2}+1} \\
\lim _{n \rightarrow \infty} C N_{\mathrm{avg}}(n)=9.0
\end{gathered}
$$

(b) Truncated Octahedron
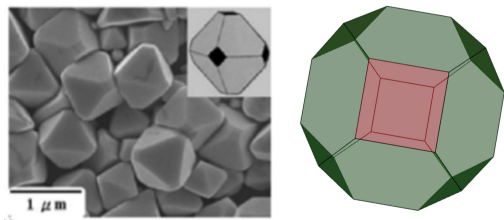

$$
\begin{gathered}
\mathrm{CN}_{\mathrm{avg}}(\mathrm{n})=\frac{6\left(22 \mathrm{n}^{2}-5 \mathrm{n}+1\right)}{15 \mathrm{n}^{2}+1} \\
\lim _{n \rightarrow \infty} C N_{\mathrm{avg}}(n)=8.8
\end{gathered}
$$

(e) Cube
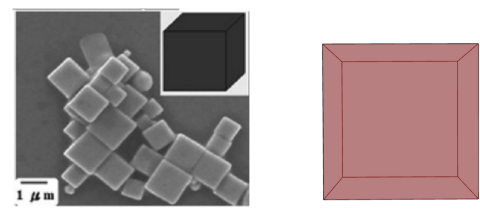

$$
\mathrm{CN}_{\mathrm{avg}}(\mathrm{n})=\frac{6\left(8 n^{2}-3 n+1\right)}{6 n^{2}+1}
$$$$
\lim _{n \rightarrow \infty} C N_{\text {avg }}(n)=8.0
$$

(h) Rhombic Dodecahedron
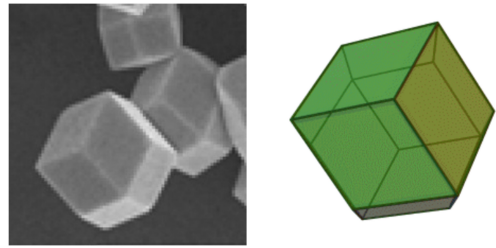

$$
\begin{gathered}
\mathrm{CN}_{\mathrm{avg}}(\mathrm{n})=\frac{6\left(7 n^{2}-2 n+1\right)}{6 n^{2}+1} \\
\lim _{n \rightarrow \infty} C N_{\text {avg }}(n)=7.0
\end{gathered}
$$

(c) Cuboctahedron
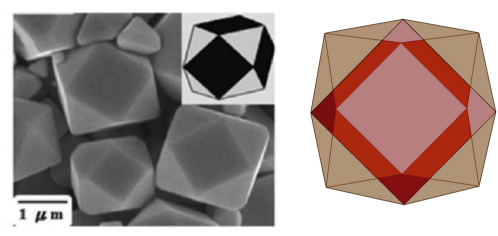

$$
\begin{gathered}
\mathrm{CN}_{\mathrm{avg}}(\mathrm{n})=\frac{6\left(7 n^{2}-3 n+1\right)}{5 n^{2}+1} \\
\lim _{n \rightarrow \infty} C N_{\mathrm{avg}}(n)=8.4
\end{gathered}
$$

(f) Decahedron
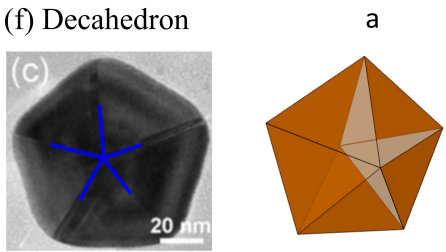

$$
\mathrm{CN}_{\mathrm{avg}}(\mathrm{n})=\frac{45 n^{2}-25 n+12}{5 n^{2}+2}
$$

$$
\lim _{n \rightarrow \infty} C N_{\text {avg }}(n)=9.0
$$

(i) Hexagonal Bipyramid
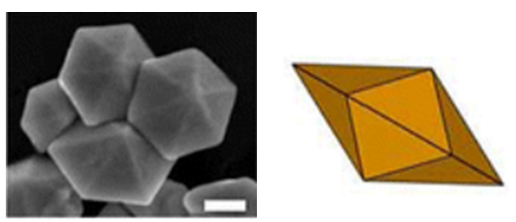

$$
\begin{gathered}
\mathrm{CN}_{\mathrm{avg}}(\mathrm{n})=\frac{3\left(17 n^{2}-7 n+2\right)}{6 n^{2}+1} \\
\lim _{n \rightarrow \infty} C N_{\mathrm{avg}}(n)=8.5
\end{gathered}
$$

Figure 8: Analytical solutions for $\mathrm{CN}_{\mathrm{avg}}(\mathrm{n})$ as a function of the number of shells, $n$, for 9 common NP structures; $n \geq 1$ in all cases. Note that for the rhombic dodecahedron, only even values of $n$ are considered. To illustrate the NP shapes, we represent cartoons generated with the code polyhedral-viewer (https://github.com/tesseralis/polyhedra-viewer). Images of corresponding NPs synthesized in experiments are also shown (left panel next to each cartoon). The experimental images are taken from the following references and are reproduced with permission. Figure 8(a): $\operatorname{Ref}^{39}$ Copyright 2014 John Wiley and Sons. Figure 8(b), (c), (d), (e) and (h): $\operatorname{Ref}^{40}$ Copyright 2010 American Chemical Society. Figure 8(f): $\operatorname{Ref}^{41}$ Copyright 2020 American Chemical Society. Figure 8(g):Ref ${ }^{42}$ Copyright 2004 John Wiley and Sons. Figure 8(i): Ref ${ }^{43}$ Copyright 2013 American Chemical Society. 


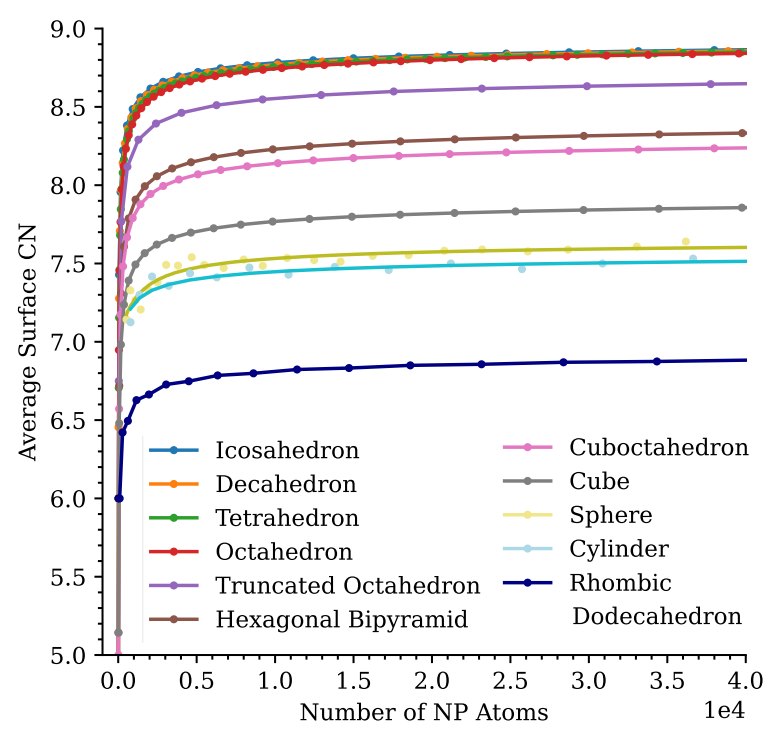

Figure 9: Dependence of $\mathrm{CN}_{\mathrm{avg}}(\mathrm{CN}<10)$ with $\mathrm{NP}$ size (in terms of number of number of atoms in the NP) for 9 FCC faceted solids, cylinders and spheres. The total number of atoms in the NP varies with the number of shells as $n^{3}$ (see section 1 in SI for partcile sizes). A spherical NP of $3 \times 10^{4}$ gold atoms has a diameter of $\sim 10 \mathrm{~nm}$. A plot with a log scale for the number of atoms is shown in Figure S17.

We used the ANA method to compute the local ITC of different NP structures and sizes and the BBA for three flat faceted surfaces (see Figure 10a). The ITC varies inversely with the average $\mathrm{CN}$. This idea can be made concrete by representing the Kapitza resistance (inverse ITC) as a function of $\mathrm{CN}_{\mathrm{avg}}(\mathrm{n})$ (see Figure 10b). The NP structures represented in the inserts highlight regions of high and low conductance. Under-coordinated atoms at vertices and edges have much higher ITCs than highly coordinated atoms located at flat surfaces. Our results show a linear correlation between Kapitza resistance and $\mathrm{CN}_{\mathrm{avg}}(\mathrm{n})$. $\mathrm{NP}$ with lower $\mathrm{CN}_{\mathrm{avg}}(\mathrm{n})$ conduct heat better than particles with high $\mathrm{CN}_{\mathrm{avg}}(\mathrm{n})$, with ratios between maximum ITC and minimum ITC of 19/11 1.7. These differences are substantial considering the particles have approximately the same average diameter $(10 \mathrm{~nm}$ ) (see SI section 1.2 for number of atoms in each particle). The results presented in Figure 9 highlight the importance of the $\mathrm{CN}$ as a variable to rationalize the ITC of particles with very complex shapes. 


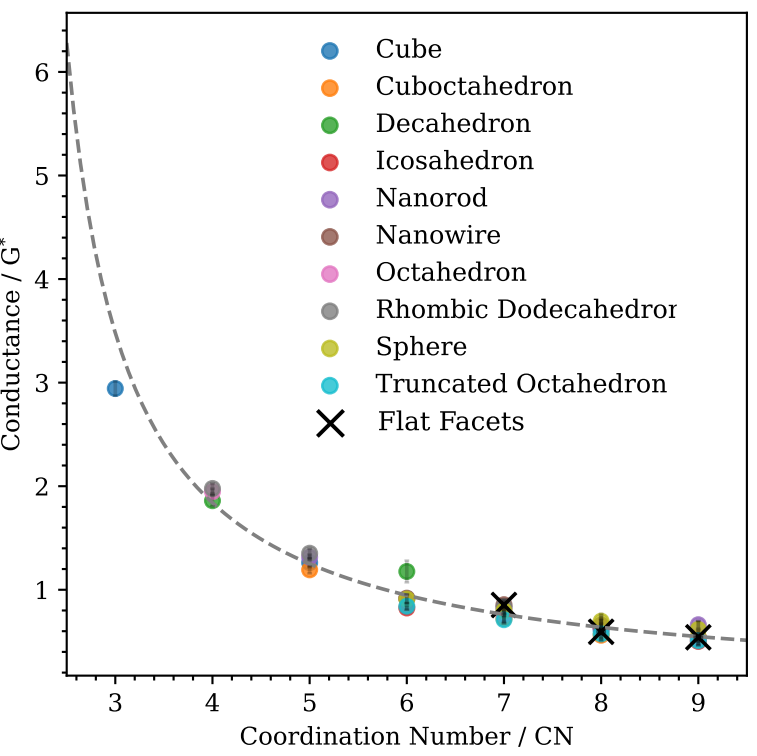

(a)

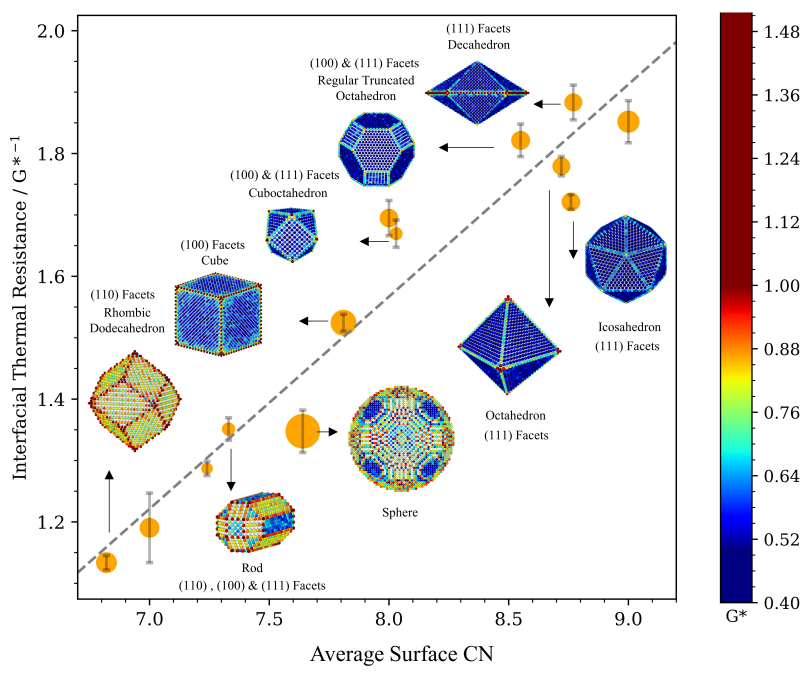

Figure 10: (Top) Variation of ITC with average coordination number, for each individual unique atomic CN across various NP shapes. (Bottom) Dependence of the Kapitza resistance with the average coordination number of the NP surface-atoms for various NP shapes. The ITC has been projected on the surface atoms for each shape, with red and orange colours representing atoms with high conductance and blue representing atoms with low ITC. The NPs used in both plots have sizes in the range of $3 \times 10^{3}$ to $3 \times 10^{4}$ atoms. The size of each point represents the number of atoms in each NP type (see SI section 1.2 for more details).

\subsection{Size dependence of the ITC}

The dependence of the ITC with particle size has attracted attention recently. ${ }^{16,17,26-28}$ Several of these studies showed an increase of the ITC with interfacial curvature or decreasing 
particle size. Interestingly, an increase in the ITC with particle size has been reported too. ${ }^{26}$ We showed in Figure 9 that the average coordination number (CN) generally increases with particle size, and in section 3.2 we established a relationship between higher thermal conductance and lower CN. These results suggest that the ITC will generally decrease with increasing particle size.

To test the size dependence of the ITC, we performed additional simulations of the octahedron, spheres, and rhombic dodecahedron (RD) nanoparticles. Our results (see Figure 11) indicate that the RD features the lowest interfacial thermal resistance (highest ITC), while the octahedron features a high thermal resistance. This general dependence is in line with the results discussed in the previous section, with the NP featuring a higher average CN showing lower ITCs. The spherical particles do also show a higher resistance for higher average CN, or with larger particle size. This result is consistent with previous observations. ${ }^{16,17,27,27,28}$

We have further explored the correlation between fluid adsorption and thermal conductance. This correlation was studied before in references, ${ }^{16,23}$ showing that the ITC increases linearly with interfacial adsorption. We calculated the adsorption using,

$$
\Gamma^{*}=\frac{N_{1}}{A^{*}}=\frac{1}{R^{* 2}} \int_{0}^{M_{1}} r^{2} \rho^{*}(r) \mathrm{d} r
$$

where $N_{1}$ is the number of particles in the first solvation shell, $A^{*}$ is the NP surface area, $\rho^{*}(r)$ is the fluid density at distance $r *$ and $M_{1}$ represents the first minimum of the density profile (see Figure S16 for radial density profiles). We obtain an approximately linear dependence between the ITC and the adsorption for the spherical particles investigated here (see Figure 11). 

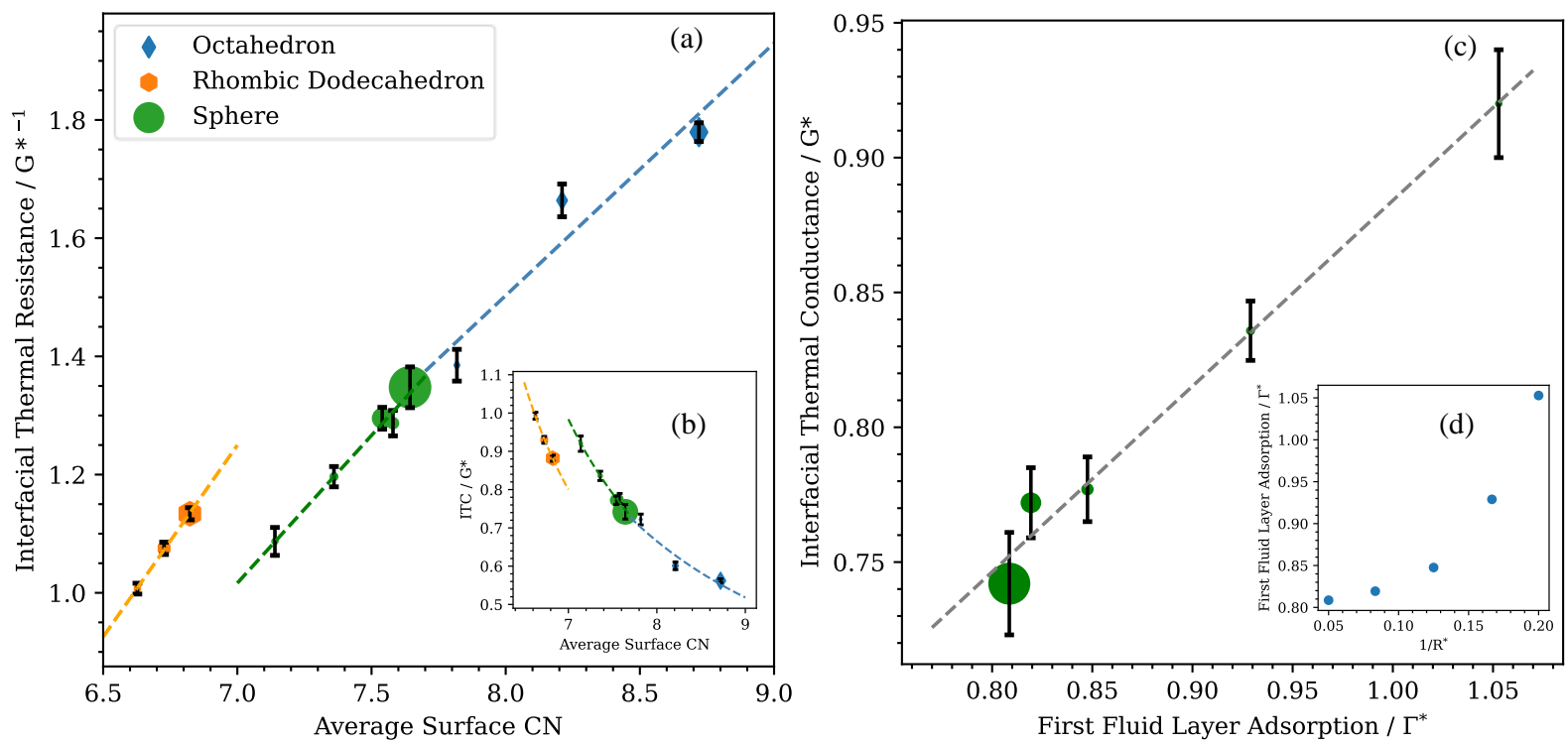

Figure 11: Dependence of the (a) Kapitza resistance and (b) ITC with the average coordination number of the NP surface-atoms for various NP shapes and sizes. The number of atoms in the NP is visualised by the marker size of each particle type. (c) Relationship between the ITC of spheres of various sizes and the adsorption of the first fluid layer (see section 1 in SI for information on particle sizes). (d) Dependence of the adsorption with the NP curvature $1 / R^{*}$ of the nanospheres.

\section{Conclusion}

We have introduced a computational approach to calculate the Interfacial Thermal Conductance (ITC) of nanomaterial-fluid interfaces. The Atomistic Nodal Approach (ANA) relies on combining "computational farming", namely using thousands of independent nonequilibrium molecular dynamics stationary simulations. We also use tools from computational geometry (KD-Trees, Voronoi polyhedra and $\alpha$-shapes) to identify surface atoms in nanoparticles and their solvation environment, which is quantified through their local coordination number. Local thermal gradients were computed via multivariate trilinear interpolation. Furthermore, the atoms in the nanomaterials are used as a nodal network to project heat fluxes, local densities and temperature gradients, and compute the interfacial thermal conductance at angstrom resolution level.

We have tested the performance of the ANA method against widely used binning meth- 
ods that partition the system of interest in well-defined geometries, such as spheres and cylinders. These geometries are amenable for theoretical treatments using the classical heat transfer theory and the heat diffusion equation. The ANA produces results entirely consistent with the binning methods, and goes beyond these methods by enabling the calculation of local properties at the atomic level. Our approach makes it possible to identify regions in the surface of the nanomaterials with contrasting ITC. Moreover, it enables us to quantify the thermal conductance of nanomaterials with complex shapes (nanocrystals with facets, vertices and edges), an in general surfaces with significant roughness, which cannot be easily mapped to standard geometries. We have applied the ANA method to nanoparticles of different shapes and sizes immersed in an atomistic fluid. The nanoparticles were heated at stationary conditions and the heat transport across the nanoparticle-fluid interface analysed using the ANA method. We conclude the following from our investigation:

- We have established a correlation between the ITC and the coordination number (CN) of atoms in the surface of the nanoparticle, where the CN quantifies the number of nearest neighbors of surface atoms with other atoms in the nanoparticle. The ITC increases with decreasing CN. Surface atoms with smaller CN share more contacts with the solvent. These contacts enhance the thermal conduction from the hot nanoparticles to the fluid. Generally, undercoordination correlates positively with higher ITC. Hence, the coordination of the surface atoms reveals itself as an approach to design nanoheaters for which the heating performance can be tuned by changing the particle geometry and modifying at the same time their ITC.

- For nanoparticles featuring facets with well-defined symmetries (111), (100), (110), the local ITC obtained for small surfaces spanning a few $\mathrm{nm}^{2}$ are very similar to the $\mathrm{CN}$ of extended infinite surfaces. This is an interesting result that provides a design principle to estimate the ITC of nanoparticles using the ITC of macroscopic surfaces. Furthermore, the ITC of atoms at edges and vertices can be obtained readily using the ANA approach, making it possible to predict the ITC of particles for a wide range of geometries. 
- Nanoparticle size and morphology act synergistically and determine the average ITC of nanoparticles. Smaller NPs have higher thermal conductances due to a more significant proportion of undercoordinated atoms at the surface. Hence, small nanoparticles are better heat conductors. Instead, larger NPs form surfaces with a higher average surface coordination number, reducing their ITC. The general dependence of the average surface coordination number with the number of NP atoms can be described using a rational expression.

- The Kapitza resistance (the inverse ITC) of 9 different nanoparticles structures varies linearly with the average CN of the surface atoms. Nanoparticles mainly consisting of (100) and (110) faces (rhombic dodecahedron and nanocubes) feature the lowest thermal resistance. Those with (111) faces (icosahedron and dodecahedron) have the highest ITC, with spherical and cylindrical geometries having intermediate conductances.

We expect the Atomistic Nodal Approach proposed here will stimulate further theoretical and experimental work to advance our understanding of thermal transport at the nanoscale and its dependence on particle size. In addition, knowledge of local ITC should be helpful to rationalize experimental observations and assist the design of nanoparticles for biomedical and, more generally, thermoplasmonic applications.

\section{Acknowledgement}

We thank the Leverhulme Trust (UK grant RPG-2018-384) for financial support, the Imperial College RCS High Performance Computing facility and the UK Materials and Molecular Modelling Hub for computational resources partially funded by the EPSRC (EP/P020194/1 and EP/T022213/1). FB acknowledges financial support from EPSRC (EP/J003859/1).

\section{Supporting Information Available}

Code and script used for generating and averaging data is publically available on github: https://github.com/bresmegroup/Atomistic_Nodal_Approach_Nanoparticles.git 
Additional data on simulation details, calculation of heat profiles, ITCs of various nanoparticles shapes, and local solvation structure of surface atoms is available in the Supporting Information.

\section{References}

(1) Hirsch, L. R.; Stafford, R. J.; Bankson, J. A.; Sershen, S. R.; Rivera, B.; Price, R. E.; Hazle, J. D.; Halas, N. J.; West, J. L. Nanoshell-mediated near-infrared thermal therapy of tumors under magnetic resonance guidance. Proceedings of the National Academy of Sciences 2003, 100, 13549-13554.

(2) Lal, S.; Clare, S. E.; Halas, N. J. Nanoshell-enabled photothermal cancer therapy: impending clinical impact. Acc Chem Res 2008, 41, 1842-1851.

(3) Pankhurst, Q. A.; Connolly, J.; Jones, S. K.; Dobson, J. TOPICAL REVIEW: Applications of magnetic nanoparticles in biomedicine. Journal of Physics D Applied Physics 2003, 36, R167-R181.

(4) Jaque, D.; Martínez Maestro, L.; del Rosal, B.; Haro-Gonzalez, P.; Benayas, A.; Plaza, J. L.; Martín Rodríguez, E.; García Solé, J. Nanoparticles for photothermal therapies. Nanoscale 2014, 6, 9494-9530.

(5) Huang, J.; Zhang, L.; Chen, B.; Ji, N.; Chen, F.; Zhang, Y.; Zhang, Z. Nanocomposites of size-controlled gold nanoparticles and graphene oxide: Formation and applications in SERS and catalysis. Nanoscale 2010, 2, 2733-2738.

(6) Govorov, A. O.; Richardson, H. H. Generating heat with metal nanoparticles. Nano today 2007, 2, 30-38.

(7) Clark, J. N.; Beitra, L.; Xiong, G.; Fritz, D. M.; Lemke, H. T.; Zhu, D.; Chollet, M.; Williams, G. J.; Messerschmidt, M. M.; Abbey, B.; Harder, R. J.; Korsunsky, A. M.; 
Wark, J. S.; Reis, D. A.; Robinson, I. K. Imaging transient melting of a nanocrystal using an X-ray laser. Proceedings of the National Academy of Sciences 2015, 112, $7444-7448$.

(8) Kirschner, M. S.; Hannah, D. C.; Diroll, B. T.; Zhang, X.; Wagner, M. J.; Hayes, D.; Chang, A. Y.; Rowland, C. E.; Lethiec, C. M.; Schatz, G. C.; Chen, L. X.; Schaller, R. D. Transient Melting and Recrystallization of Semiconductor Nanocrystals Under Multiple Electron-Hole Pair Excitation. Nano Letters 2017, 17, 5314-5320.

(9) Kirschner, M. S.; Diroll, B. T.; Brumberg, A.; Leonard, A. A.; Hannah, D. C.; Chen, L. X.; Schaller, R. D. Optical Signatures of Transiently Disordered Semiconductor Nanocrystals. ACS Nano 2018, 12, 10008-10015.

(10) Font, F.; Bresme, F. Transient Melting at the Nanoscale: A Continuum Heat Transfer and Nonequilibrium Molecular Dynamics Approach. The Journal of Physical Chemistry C 2018, 122, 17481-17489.

(11) Font, F.; Micou, W.; Bresme, F. Non-equilibrium molecular dynamics and continuum modelling of transient freezing of atomistic solids. International Journal of Heat and Mass Transfer 2021, 164, 120601.

(12) Baffou, G.; Cichos, F.; Quidant, R. Applications and challenges of thermoplasmonics. Nature Materials 2020, 19, 946-958.

(13) López-Varela, K. A.; Cayetano-Castro, N.; Kolosovas-Machuca, E. S.; Gonzalez, F. J.; Chiwo, F. S.; Rodríguez-López, J. L. Dynamic infrared thermography of nanoheaters embedded in skin-equivalent phantoms. Journal of Nanomaterials 2018, 2018.

(14) Bahadar, H.; Maqbool, F.; Niaz, K.; Abdollahi, M. Toxicity of nanoparticles and an overview of current experimental models. Iranian biomedical journal 2016, 20, 1. 
(15) Swartz, E. T.; Pohl, R. O. Thermal boundary resistance. Reviews of modern physics 1989, 61, 605 .

(16) Tascini, A. S.; Armstrong, J.; Chiavazzo, E.; Fasano, M.; Asinari, P.; Bresme, F. Thermal transport across nanoparticle-fluid interfaces: the interplay of interfacial curvature and nanoparticle-fluid interactions. Physical Chemistry Chemical Physics 2017, 19, $3244-3253$.

(17) Lervik, A.; Bresme, F.; Kjelstrup, S. Heat transfer in soft nanoscale interfaces: the influence of interface curvature. Soft Matter 2009, 5, 2407-2414.

(18) Lervik, A.; Bresme, F.; Kjelstrup, S.; Bedeaux, D.; Miguel Rubi, J. Heat transfer in protein-water interfaces. Phys. Chem. Chem. Phys. 2010, 12, 1610-1617.

(19) Rajabpour, A.; Seif, R.; Arabha, S.; Heyhat, M. M.; Merabia, S.; Hassanali, A. Thermal transport at a nanoparticle-water interface: A molecular dynamics and continuum modeling study. The Journal of chemical physics 2019, 150, 114701.

(20) Bergman, T. L.; Incropera, F. P.; DeWitt, D. P.; Lavine, A. S. Fundamentals of heat and mass transfer; John Wiley \& Sons, 2011.

(21) Shenogina, N.; Godawat, R.; Keblinski, P.; Garde, S. How wetting and adhesion affect thermal conductance of a range of hydrophobic to hydrophilic aqueous interfaces. Physical review letters 2009, 102, 156101.

(22) Patel, H. A.; Garde, S.; Keblinski, P. Thermal Resistance of Nanoscopic Liquid-Liquid Interfaces: Dependence on Chemistry and Molecular Architecture. Nano Letters 2005, $5,2225-2231$.

(23) Muscatello, J.; Chacón, E.; Tarazona, P.; Bresme, F. Deconstructing Temperature Gradients across Fluid Interfaces: The Structural Origin of the Thermal Resistance of Liquid-Vapor Interfaces. Phys. Rev. Lett. 2017, 119, 045901. 
(24) Simon, J.-M.; Kjelstrup, S.; Bedeaux, D.; Hafskjold, B. Thermal Flux through a Surface of n-Octane. A Non-equilibrium Molecular Dynamics Study. The Journal of Physical Chemistry B 2004, 108, 7186-7195.

(25) Merabia, S.; Shenogin, S.; Joly, L.; Keblinski, P.; Barrat, J.-L. Heat transfer from nanoparticles: A corresponding state analysis. Proceedings of the National Academy of Sciences 2009, 106, 15113-15118.

(26) Neidhart, S. M.; Gezelter, J. D. Thermal transport is influenced by nanoparticle morphology: A molecular dynamics study. The Journal of Physical Chemistry C 2018, 122, 1430-1436.

(27) Hu, M.; Poulikakos, D.; Grigoropoulos, C. P.; Pan, H. Recrystallization of picosecond laser-melted $\mathrm{ZnO}$ nanoparticles in a liquid: A molecular dynamics study. The Journal of Chemical Physics 2010, 132, 164504.

(28) Wilhelmsen, Ø.; Bedeaux, D.; Kjelstrup, S. Heat and mass transfer through interfaces of nanosized bubbles/droplets: the influence of interface curvature. Phys. Chem. Chem. Phys. 2014, 16, 10573-10586.

(29) Heinz, H.; Vaia, R. A.; Farmer, B. L.; Naik, R. R. Accurate Simulation of Surfaces and Interfaces of Face-Centered Cubic Metals Using 12-6 and 9-6 Lennard-Jones Potentials. The Journal of Physical Chemistry C 2008, 112, 17281-17290.

(30) Irving, J. H.; Kirkwood, J. G. The Statistical Mechanical Theory of Transport Processes. IV. The Equations of Hydrodynamics. The Journal of Chemical Physics 1950, $18,817-829$.

(31) Plimpton, S. Fast parallel algorithms for short-range molecular dynamics. Journal of computational physics 1995, 117, 1-19. 
(32) LAMMPS Molecular Dynamics Simulator, howpublished = https://www.lammps . org/index.html, note $=$ Accessed: 2021-07-14.

(33) Gardiner, J. D.; Behnsen, J.; Brassey, C. A. Alpha shapes: determining 3D shape complexity across morphologically diverse structures. BMC evolutionary biology $\mathbf{2 0 1 8}$, $18,1-16$.

(34) Edelsbrunner, H.; Mücke, E. P. Three-dimensional alpha shapes. ACM Transactions on Graphics (TOG) 1994, 13, 43-72.

(35) Maneewongvatana, S.; Mount, D. M. Analysis of approximate nearest neighbor searching with clustered point sets. Data Structures, Near Neighbor Searches, and Methodology 2002, 59, 105-123.

(36) Ismail, A. E.; Grest, G. S.; Stevens, M. J. Capillary waves at the liquid-vapor interface and the surface tension of water. The Journal of chemical physics 2006, 125, 014702.

(37) Espinosa, A.; Reguera, J.; Curcio, A.; Muñoz-Noval, Á.; Kuttner, C.; Van de Walle, A.; Liz-Marzán, L. M.; Wilhelm, C. Janus Magnetic-Plasmonic Nanoparticles for Magnetically Guided and Thermally Activated Cancer Therapy. Small 2020, 16, 1904960.

(38) Kaatz, F. H.; Bultheel, A. Magic mathematical relationships for nanoclusters. Nanoscale research letters 2019, 14, 1-12.

(39) Zheng, Y.; Liu, W.; Lv, T.; Luo, M.; Hu, H.; Lu, P.; Choi, S.-I.; Zhang, C.; Tao, J.; Zhu, Y., et al. Seed-Mediated Synthesis of Gold Tetrahedra in High Purity and with Tunable, Well-Controlled Sizes. Chemistry-An Asian Journal 2014, 9, 2635-2640.

(40) Hsu, S.; Su, P. Y.; Jian, L.; Chang, A.; Lin, I. J. Vertex and Edge Truncated Octahedron Gold Crystals. N-alkylimidazole and Silver (I) Ion Controlled Morphology Transformation. Inorganic chemistry 2010, 49, 4149-4155. 
(41) Ma, X.; Lin, F.; Chen, X.; Jin, C. Unveiling Growth Pathways of Multiply Twinned Gold Nanoparticles by In Situ Liquid Cell Transmission Electron Microscopy. ACS nano 2020, 14, 9594-9604.

(42) Kim, F.; Connor, S.; Song, H.; Kuykendall, T.; Yang, P. Platonic gold nanocrystals. Angewandte Chemie International Edition 2004, 43, 3673-3677.

(43) Personick, M. L.; Langille, M. R.; Wu, J.; Mirkin, C. A. Synthesis of gold hexagonal bipyramids directed by planar-twinned silver triangular nanoprisms. Journal of the American Chemical Society 2013, 135, 3800-3803. 


\section{Graphical TOC Entry}

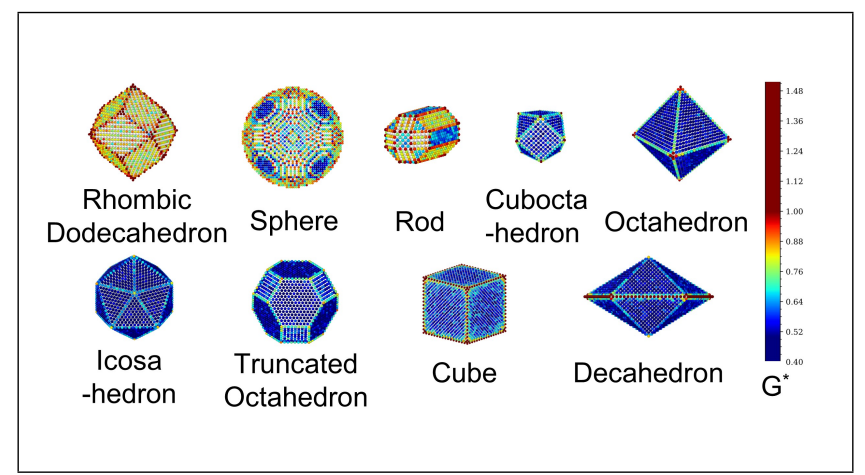

